\title{
New automated image analysis method for the assessment of Ki-67 labeling index in meningiomas
}

\author{
Bartlomiej Grala1, Tomasz Markiewicz ${ }^{1,2}$, Wojciech Kozlowski', \\ Stanisław Osowski ${ }^{2,3}$, Janina Słodkowska1, Wielisław Papierz ${ }^{4}$ \\ ${ }^{1}$ Department of Pathology, Military Institute of Health Services, Warsaw, Poland \\ ${ }^{2}$ Warsaw University of Technology, Warsaw, Poland \\ ${ }^{3}$ Military University of Technology, Warsaw, Poland \\ ${ }^{4}$ Pathomorphology Department, Medical University of Lodz, Lodz, Poland
}

\begin{abstract}
Many studies have emphasised the importance of Ki-67 labeling index (LI) as the proliferation marker in meningiomas. Several authors confirmed, that Ki-67 LI has prognostic significance and correlates with likelihood of tumour recurrences. These observations were widely accepted by pathologists, but up till now no standard method for Ki-67 LI assessment was developed and introduced for the diagnostic pathology. In this paper we present a new computerised system for automated Ki-67 LI estimation in meningiomas as an aid for histological grading of meningiomas and potential standard method of Ki-67 LI assessment. We also discuss the concordance of Ki-67 LI results obtained by presented computerized system and expert pathologist, as well as possible pitfalls and mistakes in automated counting of immunopositive or negative cells. For the quantitative evaluation of digital images of meningiomas the designed software uses an algorithm based on mathematical description of cell morphology. This solution acts together with the Support Vector Machine (SVM) used in the classification mode for the recognition of immunoreactivity of cells. The applied sequential thresholding simulated well the human process of cell recognition. The same digital images of randomly selected tumour areas were parallelly analysed by computer and blindly by two expert pathologists. Ki-67 labeling indices were estimated and the results compared. The mean relative discrepancy between the levels of Ki-67 LI by our system and by the human expert did not exceed $14 \%$ in all investigated cases. These preliminary results suggest that the designed software could be an useful tool supporting the diagnostic digital pathology. However, more extended studies are needed for approval of this suggestion.
\end{abstract}

Keywords: meningioma, immunohistochemistry, Ki-67, labeling index, Support Vector Machine, sequential thresholding

\section{Introduction}

Meningiomas belong to the most frequent primary intracranial tumours, of which one on four appears to be a meningothelial cell neoplasm [1]. A vast majority of meningiomas are considered as benign tumours and are assigned to histologic grade I (according to the WHO classification of the central nervous system tumours) [1]. About 5-7\% of meningothelial cell tumours are of greater likelihood of recurrences and/or more aggressive biological behaviour. These tumours form a group of atypical meningiomas which is graded as II. Only $1-2 \%$ of meningiomas belong to grade III and are recognized as the anaplastic meningiomas

Correspondence: B. Grala, Department of Pathology, Military Institute of Health Services, 128 Szaserów Str., 00-909 Warsaw, Poland; e-mail: grallab@esculap.pl with evident features of malignancy e.g.: anaplasia, enormously high mitotic activity and median survival below 2 years $[1,2]$.

A serious clinical problem remain recurrences of the resected neoplasms. Atypical and anaplastic meningiomas tend to give recurrences in $29-40 \%$ and $50-78 \%$, respectively. However $7-20 \%$ of 'typical' grade I meningiomas give recurrences as well [1]. Therefore, the correct diagnosis of high grades meningiomas is of great importance even though the WHO classification gives only vague hints for the differential diagnosis of grade I and II [1].

Many investigators have searched for the reliable predictive markers for meningothelial cell tumours [3]. Among several accepted proliferative markers Ki-67 labeling index (LI) is a useful one in prediction of the biological behavior and growth of human tumours, including various neoplasms of the central nervous 
system $[3,4]$. The Ki-67 antigen is detected in the cell nucleus almost an active phases of mitotic cell division, that is: $G_{1}, S, G_{2}$, except $G_{0}$.

In many studies on meningiomas the significant correlation was confirmed between tumour grades and Ki-67 LI. Different laboratories estimated Ki-67 mean indices as $0.9-3.8 \%, 3.3-9.9 \%$ and $8.7-14.7 \%$, for meningiomas of grade I, II and III, respectively $[1,5,6]$. The differences in Ki-67 LI for one histological grade can result from the various methods applied for Ki-67 LI quantisation $[2,6,7]$. Also, various groups of investigators postulated the different cut-off values of Ki-67 LI related significantly to the increased risk of recurrences. Dependly on the studies and used methods of Ki-67 LI counting the index ranged from 2 to 4\% [1,7]. Lack of consensus for standardised procedure of Ki-67 LI estimation makes any clear interpretation of the published results impossible. Moreover, the researchers are unable to perform reliable broad meta-analytic studies.

In our preliminary study the recently designed computer software was implemented for automated assessment of Ki-67 LI in meningiomas. The results of automated quantisation of Ki-67 LI showed high concordance with the results of manual Ki-67 LI assessment. The obtained data are encouraging for the extended investigations on the validation of this computerised method for the routine neuropathological diagnostics as a new standard in Ki67 LI estimation in meningiomas.

\section{Materials and methods}

Thirty seven cases of meningiomas were randomly selected from the archives of the Pathology Department of the Military Institute of Health Services (Warsaw, Poland) and from the Pathomorphology Department of the Medical University of Lodz (Poland). The postoperative material included: 11 cases of meningothelial meningiomas (WHO grade I), 20 cases of atypical meningiomas (WHO grade II) and 6 cases of the anaplastic type (WHO grade III). For the histological typing and grading of the tumours the criteria of WHO classification were used [1]. In the group of atypical meningiomas two subgroups were distinguished: 'IIA' and 'IIB', which encountered 12 and 8 cases, respectively. As the morphological criterion for the subgroup IIA the mitotic index exceeding 3 mitoses per 10 high-power fields (HPF: 400x magnification) was used. The atypical meningiomas were classified to the subgroup IIB on the base of the mitotic indices below 4/10HPF and the additional morphological criteria proposed by the WHO classification, which is the presence of at least three of morphological features such as: $1 /$ increased cellularity of tumour, 2/prominent nucleoli, $3 /$ small cells with high nuclear and cytoplasmic ratio, 4/ patternless or sheet-like appearance, $5 /$ focal geographic or spontaneous necrosis [1].

The histological paraffin slides of each meningioma underwent standard immunohistochemical staining with monoclonal mouse anti-human Ki-67 antibody (clone MIB-1; manufacturer: Dako, Denmark; product no. M7240), in dilution of 1:100. Dako's EnVision $^{\mathrm{TM}}$ (product no. K4001) was used as the visualisation system.

For the digital images acquisition ten microscopic areas were randomly selected from each slide. The selection was performed with the Olympus DX-50 microscope at 400x magnification. The Olympus Camedia digital camera working in red-green-blue (RGB) format and resolution $576 \times 768$ was used for the images prepara- tion. Total number of 370 digital images underwent the investigation. Each digital image covered field of $320 \times 240 \mu \mathrm{m}$ with the mean number of neoplastic cells as 652 (ranging from 306 to 1039).

The following procedure was the images analysis based on the recently developed computerised automated system for cell counting. Our system significantly differs from other known systems, by the nuclei extraction algorithm which simulates the human sight strategy used in recognition of separated cellular nuclei. The operations used by the system included: the sequential thresholding, filtering, watershed algorithm and other standard morphological operations for the extraction and the counting of tumor cells $[8,9]$. The sequential thresholding system cooperated with the Support Vector Machine (SVM) $[10,11]$ that worked in the classification mode. SVM was also used in the final stage of the algorithm after thresholding for the recognition of two types of cells: the immunopositive and immunonegative cells.

The main task in the process of image analysis was to extract all cells and identify them as immunopositive or immunonegative. Many solutions were used to solve these problems in various image-processing methods, but they were not always accurate [12$16]$. We proposed a model which combines the mathematical morphology methods and the SVM $[10,11]$. SVM is well known as an excellent and efficient classification tool used in the recognition process. In our study SVM was used in two steps for: 1/construction of maps of pixels belonged to the stained (brown) and unstained (blue) nuclei classes; and 2/recognition of cells immunopositivity.

In order to use the SVM network we had to select the learning data and precisely define examples of the recognised classes of pixels. For the learning purpose we used the manually selected data containing 100 pixels belonging to the blue cell nuclei (the first class), 100 pixels of the brown cell nuclei (the second class) and 100 pixels of the background (the third class). In this way we defined the data samples that represented three classes of image pixels under recognition. Because the SVM classifier is capable to identify two classes at once, we used three SVM networks. By this way each SVM network recognized one combination of two classes arranged in one-against-one strategy [10]. In the first step of our solution, we used the linear kernel SVM to perform the following mathematical operation [10]:

$$
\text { l) }(\mathbf{x})=\mathbf{w}^{T} \mathbf{x}+b
$$

where $\mathrm{x}$ is an input vector containing three RGB components of the pixel, $\mathrm{w}$ is the weight vector and $b$ is the bias. In the learning stage $\mathrm{D}(\mathrm{x})$ is associated with 1 for the first class and -1 for the alternative class for any combination of classes. The learning process was based on the maximization of the margin separating two different classes. This provided a minimum amount of recognition errors. The learning process was followed by transformation of that primary problem into the dual problem, which represented a quadratic programming task $[10,11]$.

After the learning stage the vector $\mathrm{w}$ and bias value $b$ were fixed. Next we calculated the appropriate $\mathrm{D}(\mathrm{x})$ value for each pixel of an image, characterized by the 3-dimensional vector $\mathrm{x}$. Dependently on the specific value of $\mathrm{D}(\mathrm{x})$, the SVM system classified the analyzed pixels into the groups of blue or brown cell nuclei or to the background. The higher $\mathrm{D}(\mathrm{x})$ was, the greater was probability that the pixel $\mathrm{x}$ belonged to the appropriate cell nuclei group. Next that value was used as the input data for the sequential thresholding method (STM). Here the STM performed the following mathematical operations:

$$
T_{i}(L(\mathbf{x}))=\left\{\begin{array}{lll}
1 & \text { if } \\
0 & \text { clsc }
\end{array}\right.
$$

where $\mathrm{D}(\mathrm{x})$ represents the SVM output signal determined for each pixel $\mathrm{x}$ of the original image and $\mathrm{t}$ is the actual bias. The STM starts from $t=\min (D(x))$ and then the $t$ value increases step by step until 


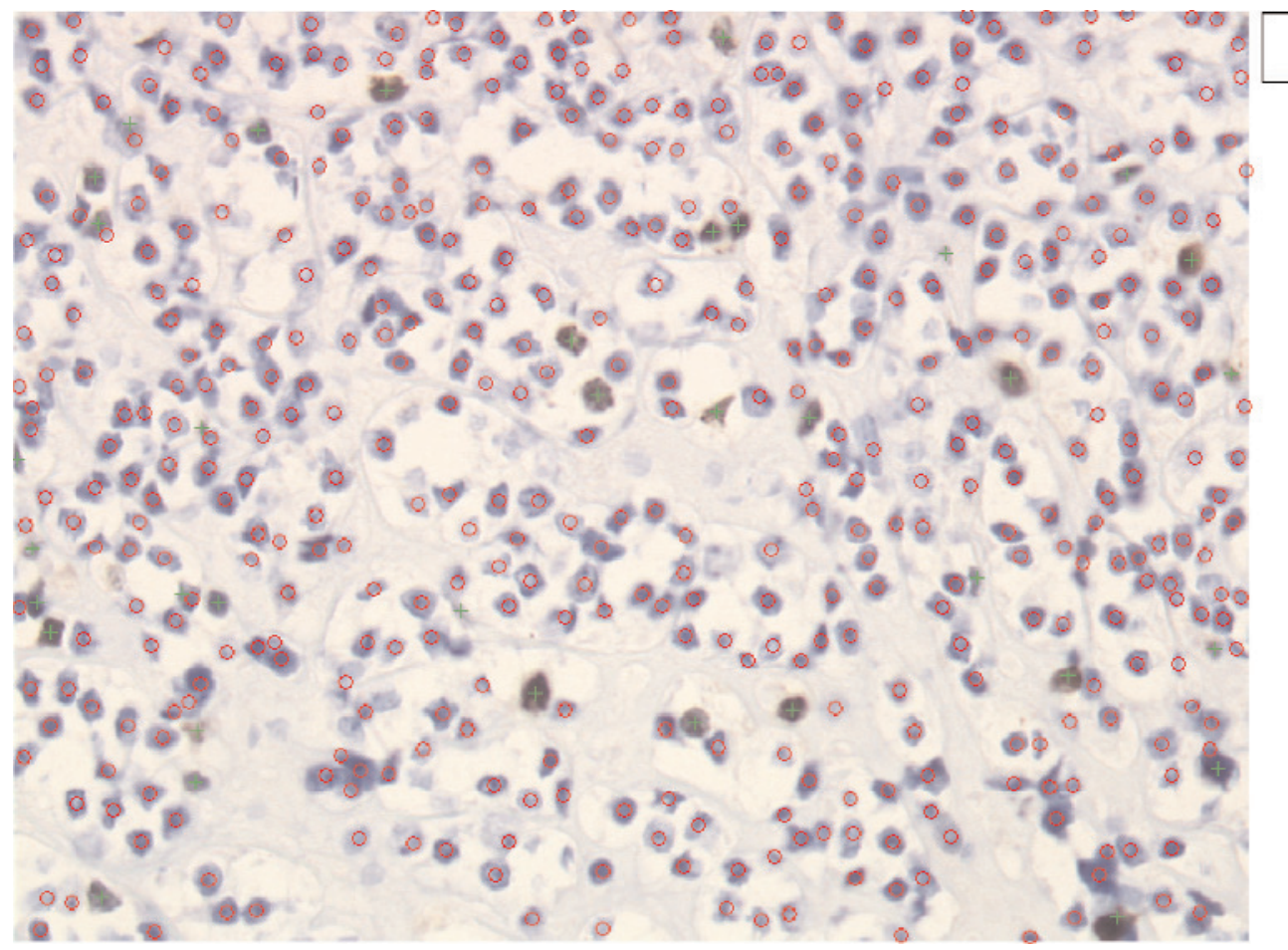

$-92.7 \%, 460$
+ $+7.3 \%, 36$

Fig. 1. Ki-67 labeling index evaluation in digital image of atypical meningioma. The system marked immunonegative cells with circles, and immunopositive with crosses. Ki-67 LI result is presented in the upper-right corner.

it reaches the maximum $\mathrm{D}(\mathrm{x})$. All compact objects separated by the thresholding operations and fulfilling the defined cellular nuclei area restriction are added to the cell nuclei mask. That is how we extracted most of the recognizable cells. The additional step of the watershed algorithm application was used to perform some corrections aimed to separate the adjacent or overlapping nuclei [8].

The next step of the algorithm was a separation of the extracted nuclei into two groups: the immunopositive (i.e. brown group); immunonegative (i.e. blue group). The SVM classifier of the Gaussian kernel, which was programmed for the nuclei pixels recognition accordingly to color of its component, also can be used for such task. If the majority of pixels in the cell nucleus were found as brown, all pixels forming the extracted nucleus were classified as immunopositive. In such case, the cell nucleus was added to the immunopositive nuclei mask. If the majority of pixels in the cell nucleus were blue, it was added to the immunonegative nuclei mask. The final, simple task for the Matlab program [9] was counting of the separated nuclei gathered in the respective masks: immunopositive or immunonegative. The described multistep procedure was applied in our analysis of 370 images. Figure 1 presents an example of atypical meningioma proceeded by our software.

Parallelly to the automated procedure, the manual estimation of $\mathrm{Ki}-67$ indices was performed blindly by two experienced pathologists for all analysed by the digital image system. The evaluation of the designed software was carried on in the comparative analysis of the results obtained in the manual and automated computerized method.

\section{Results}

The final Ki-67 labeling index was calculated as the mean value of the $\mathrm{Ki}-67 \mathrm{LI}$ obtained from ten images of each tumour case. The measurement was done by manual reading and by automated computerized system. The Ki-67 LI results obtained by both methods are presented in Table 1.

The mean values for Ki-67 LI in automated and manual method showed very high accordance in all studied cases of 3 categories of the histological grades. For all analysed cases mean Ki-67 labeling index estimated manually was $5.84 \%$, and $5.98 \%$ when automated method was applied. For meningiomas of grade I mean Ki-67 LI was $1.85 \%$ in manual counting and $1.91 \%$ by automated method. For the whole group of atypical meningiomas mean indices were 5.19\% (counted manually) and 5.29\% (computerised counting). For the subgroup 'IIA' the results were $7.89 \%$ and $7.97 \%$, and for subgroup 'IIB' $-2.54 \%$ and $2.61 \%$, respectively. In anaplastic meningiomas the manual counting gave $11.13 \%$ as mean $\mathrm{Ki}-67 \mathrm{LI}$ and the automated method $-11.42 \%$. For the individual cases the relative discrepancies between Ki-67 LI estimated manually and by the computerised system were very low. In the group of meningothelial meningiomas it ranged from 0 to $14 \%$ with mean value as $4.64 \%$. In the subgroup 'IIA' it ranged from 0 to $9 \%$, mean $2.17 \%$; in the subgroup 'IIB' - from 0 to $5 \%$, mean $2.5 \%$. For the anaplastic tumours the range of relative discrepancy was from 0 to $8 \%$, with mean value as $3.67 \%$. For all cases the mean discrepancy between results of manual and automated Ki-67 LI counting was $3.21 \%$.

\section{Discussion}

The high concordance of Ki-67 LI results obtained by manual and automated quantisation indicates that the designed system produces very limited number of counting errors. Table 2 depicts numerical results of the analysis in 12 samples of images and summarises 
Table 1. Comparison of Ki-67 labeling indices estimated by computerized system and by manual reading of the pathologist.

\begin{tabular}{|c|c|c|c|c|}
\hline \multirow{2}{*}{$\begin{array}{l}\text { Case } \\
\text { No. }\end{array}$} & \multirow[b]{2}{*}{ Grade } & \multicolumn{2}{|c|}{ Ki 67 LI (\%) } & \multirow{2}{*}{$\begin{array}{c}\text { Relative } \\
\text { Discrepancy } \\
\%\end{array}$} \\
\hline & & $\begin{array}{c}\text { Manual } \\
\text { counting }\end{array}$ & $\begin{array}{l}\text { Automated } \\
\text { counting }\end{array}$ & \\
\hline 1 & I & 0.58 & 0.56 & 4 \\
\hline 2 & I & 0.79 & 0.81 & 2 \\
\hline 3 & I & 0.79 & 0.84 & 6 \\
\hline 4 & I & 0.83 & 0.94 & 13 \\
\hline 5 & I & 1.64 & 1.69 & 3 \\
\hline 6 & I & 2.01 & 2.02 & 0 \\
\hline 7 & I & 2.2 & 2.29 & 4 \\
\hline 8 & I & 2.46 & 2.43 & 2 \\
\hline 9 & I & 2.76 & 2.74 & 1 \\
\hline 10 & I & 2.97 & 3.39 & 14 \\
\hline 11 & I & 3.32 & 3.28 & 2 \\
\hline \multicolumn{2}{|c|}{ Mean } & 1.85 & 1.91 & 4.64 \\
\hline 12 & $\Pi \Lambda^{*}$ & 0.95 & 1.04 & 9 \\
\hline 13 & IIA* & 2.76 & 2.84 & 2 \\
\hline 14 & IA* & 4.95 & 4.97 & 0 \\
\hline 15 & $\Pi A^{*}$ & 5.49 & 5.58 & 1 \\
\hline 16 & $\Pi \mathrm{A}^{*}$ & 5.98 & 6.07 & 1 \\
\hline 17 & $\Pi A^{*}$ & 6.3 & 6.32 & 0 \\
\hline 18 & IIA* & 6.48 & 6.59 & 1 \\
\hline 19 & IA* & 9.08 & 9.04 & 1 \\
\hline 20 & IIA* & 9.16 & 9.22 & 0 \\
\hline 21 & ШA* & 9.59 & 10.08 & 5 \\
\hline 22 & $\Pi \mathrm{A}^{*}$ & 14.29 & 13.98 & 3 \\
\hline 23 & $\Pi \Lambda^{*}$ & 19.21 & 19.96 & 3 \\
\hline \multicolumn{2}{|c|}{ Mean } & 7.85 & 7.97 & 2.17 \\
\hline 24 & IIB* & 0.68 & 0.69 & 1 \\
\hline 25 & $\mathrm{IIB}^{*}$ & 1.25 & 1.29 & 3 \\
\hline 26 & IIB* & 1.42 & 1.46 & 2 \\
\hline 27 & $\mathrm{IIB}^{*}$ & 1.78 & 1.85 & 3 \\
\hline 28 & IIB* & 2.28 & 2.37 & 3 \\
\hline 29 & IIB* & 3.59 & 3.58 & 1 \\
\hline 30 & $\mathrm{IIB}^{*}$ & 4.39 & 4.5 & 2 \\
\hline 31 & $\mathrm{IIB}^{*}$ & 4.89 & 5.14 & 5 \\
\hline \multicolumn{2}{|c|}{ Mean } & 2.54 & 2.61 & 2.5 \\
\hline 32 & III & 6.67 & 7.14 & 7 \\
\hline 33 & III & 8.98 & 9.75 & 8 \\
\hline 34 & III & 9.71 & 9.83 & 1 \\
\hline 35 & III & 12.39 & 12.94 & 4 \\
\hline 36 & III & 14.1 & 13.89 & 2 \\
\hline 37 & III & 14.9 & 14.99 & 0 \\
\hline \multicolumn{2}{|c|}{ Mean } & 11.13 & 11.42 & 3.67 \\
\hline
\end{tabular}

the mistakes done by the automated system in cell nuclei counting and recognition.
Several types of errors can be done by the automated system such as:

1. Failed segmentation of cluster of immunonegative nuclei.

2. Failed extraction of immunonegative nucleus from the background.

3. Counting the part of immunonegative or positive nucleus as another immunonegative.

4. Failed segmentation of cluster of immunopositive nuclei

5. Failed extraction of immunopositive nucleus from the background.

6. Counting the part of immunonegative or positive nucleus as another immunopositive.

7. Counting the immunopositive nucleus as immunonegative.

8. Counting the immunonegative nucleus as immunopositive.

9. Counting the part of background as immunonegative nucleus.

10.Counting the part of background as immunopositive nucleus.

Generally these errors can be divided in five categories, depending on their effect on final result:

A) Underestimation of immunonegative nuclei - error types 1 and 2.

B) Overestimation of immunonegative nuclei - error types 3 and 9.

C) Underestimation of immunopositive nuclei - error types 4 and 5 .

D) Overestimation of immunopositive nuclei - error types 6 and 10 .

E) Adverse changes in estimation of both immunonegative and immunopositive nuclei - error types 7 and 8.

In our study the dominating errors were of type 1 , 2 and 3. They reflect difficulties of the system in extracting the immunonegative nuclei from the background and separating them when they are grouped in clusters. Opposite, the errors affecting estimation of immunopositive nuclei were extremely rare in tumour areas with Ki-67 labeling indices lower than 15\%.

In 12 sample images depicted in Table 2 additional analysis of possible range of Ki-67 labeling indices was performed. MIN LI and MAX LI were estimated as possible minimal and maximal $\mathrm{Ki}-67$ labeling indices. When the MIN LI value was calculated, the quantity of immunonegative nuclei was taken as the sum of nuclei actually recognized as negative by automatic system and the sum of truly negative nuclei omitted by it. The quantity of immunopositive nuclei was estimated to be the difference between the actual results given by the system and the sum of falsely recognized positive nuclei. Analogically, in calculation of MAX LI value the quantity of the immunonegative nuclei was estimated from the difference between the 
Table 2. The detailed numeric analysis of twelve images carried on by the automated system. Neg - number of immunonegative nuclei, Pos - number of immunopositive nuclei; MAX LI - possible highest Ki-67 LI value, MIN LI - possible lowest Ki-67 LI value.

\begin{tabular}{|c|c|c|c|c|c|c|c|c|c|c|c|c|c|c|c|c|c|c|}
\hline Image & \multicolumn{3}{|c|}{ Manual reading } & \multicolumn{1}{|c|}{ Automated reading } & \multicolumn{10}{|c|}{ Index of specific error (see text) } & \multicolumn{1}{c|}{$\begin{array}{c}\text { Max } \\
\text { LI }\end{array}$} & $\begin{array}{c}\text { Min } \\
\text { LI }\end{array}$ \\
\hline & Neg & Pos & $\begin{array}{r}\text { Ki-67 } \\
\text { LI (\%) }\end{array}$ & Neg & Pos & $\begin{array}{c}\text { Ki-67 } \\
\text { LI (\%) }\end{array}$ & 1 & 2 & 3 & 4 & 5 & 6 & 7 & 8 & 9 & 10 & $\%$ & $\%$ \\
\hline 1 & 890 & 4 & 0,45 & 855 & 4 & 0,47 & 51 & 8 & 3 & 0 & 0 & 0 & 0 & 0 & 21 & 0 & 0,48 & 0,44 \\
\hline 2 & 511 & 8 & 1,54 & 530 & 8 & 1,49 & 16 & 4 & 38 & 0 & 0 & 0 & 0 & 0 & 1 & 0 & 1,60 & 1,43 \\
\hline 3 & 532 & 16 & 2,92 & 515 & 15 & 2,83 & 37 & 3 & 3 & 0 & 0 & 1 & 2 & 0 & 18 & 0 & 3,34 & 2,46 \\
\hline 4 & 777 & 31 & 3,84 & 754 & 30 & 3,83 & 31 & 6 & 4 & 0 & 0 & 0 & 1 & 0 & 9 & 0 & 4,02 & 3,65 \\
\hline 5 & 433 & 27 & 5,87 & 438 & 25 & 5,40 & 9 & 4 & 16 & 0 & 1 & 0 & 1 & 0 & 1 & 0 & 6,04 & 5,25 \\
\hline 6 & 723 & 66 & 8,37 & 723 & 65 & 8,25 & 20 & 2 & 19 & 0 & 0 & 1 & 2 & 0 & 1 & 0 & 8,72 & 7,91 \\
\hline 7 & 419 & 63 & 13,07 & 454 & 65 & 12,52 & 2 & 4 & 40 & 0 & 0 & 1 & 0 & 1 & 2 & 0 & 13,63 & 12,02 \\
\hline 8 & 298 & 52 & 14,86 & 293 & 52 & 15,07 & 6 & 14 & 14 & 0 & 0 & 1 & 1 & 0 & 0 & 0 & 16,01 & 14,01 \\
\hline 9 & 546 & 97 & 15,09 & 544 & 96 & 15,00 & 19 & 7 & 20 & 0 & 2 & 1 & 3 & 2 & 3 & 1 & 16,32 & 13,86 \\
\hline 10 & 345 & 75 & 17,86 & 340 & 77 & 18,47 & 9 & 21 & 19 & 2 & 3 & 12 & 5 & 0 & 1 & 0 & 21,64 & 14,94 \\
\hline 11 & 535 & 169 & 24,01 & 527 & 164 & 23,73 & 38 & 8 & 21 & 10 & 0 & 5 & 0 & 0 & 17 & 0 & 26,24 & 21,72 \\
\hline 12 & 265 & 103 & 27,99 & 262 & 98 & 27,22 & 5 & 13 & 12 & 8 & 1 & 6 & 2 & 0 & 1 & 0 & 30,62 & 24,7 \\
\hline
\end{tabular}

number of negative nuclei actually recognized by the system and the total number of falsely recognized negative nuclei. The quantity of immunopositive nuclei was the sum of the positive nuclei given by automatic system and other truly immunopositive nuclei that were not recognized by the system. Thus the MIN and MAX LI measurements represented the predicted worst cases of estimation by the computerised system. The greater MIN-MAX LI range, more possible was a mistake in Ki-67 index assessment. The results presented in the Table 2 indicate that the analysed MINMAX LI ranges gradually increased with elevating LI. However, significantly greater ranges appeared at LI over $15 \%$, related mainly to anaplastic meningiomas, what seems to be of little clinical importance.

To summarise, proliferative activity of meningiomas measured by Ki-67 labeling index correlates with many parameters, not only with the histological grade of tumour. Many studies confirmed the importance of tumour localisation, proliferative markers like $\mathrm{Ki}-67$, the type of tumour resection and also the specific genetic alterations, for the risk of neoplasm recurrences $[1,6,17,18]$.

Some attempt have been made in the standardization of the Ki-67 LI counting procedures [19]. However, still no widely accepted Ki-67 counting method was developed [2]. The problem of different counting procedures was exhaustively described by Nakasu et al. [7]. The authors of this paper compared two methods of Ki-67 LI assessment - counting of Ki-67 positive cells in the area with the highest labeling (HL method) versus counting the cells in randomly selected areas (RS method). The authors stated that although the RS method needs more microscopic fields for evaluation than the HL method, and requires good quality specimens, it provides a better prediction for meningiomas growth and recurrence. Thus, in our study, we chose the RS method for the microscopic digital images analysis.

The results of our preliminary study on Ki-67 LI on 37 meningiomas of grades I - III, carried on with the new automated computerised system gave the data encouraging for the further validation of the method. The described analysis of the digital images implemented the algorithm which based on mathematical description of cell morphology, combined with the SVM seems to be an efficient tool for the classification of nuclear immunoreactivity.

The results of the cell recognition achieved by the designed automated system showed great accordance with the results carried on by the manual method. A few errors in the automated cell counting might be related to the inaccurate recognition of the immunonegative nuclei by the system. The main types of noted errors were 1 / failure to segmentation of cluster of immunonegative nuclei, 2/ failure to extraction of immunonegative nucleus from the background, 3/ counting the part of immunonegative or positive nucleus as another immunonegative, and, at lower degree, 4/ counting the part of background as immunonegative nucleus. The reasons of these errors could be: a/ weak hematoxyllin staining of the slide, $b$ / similarity of the immunonegative nuclei to nonneoplastic cell nuclei present in the background, c/ merging of many cells together because of their close proximity to each other. Nevertheless, the MIN LI and 
MAX LI values (Table 2) were only slightly different from the manually estimated Ki-67 levels, confirming the satisfactory solution achieved by our automated system.

The results of our investigation suggest that developed system could be an alternative solution for the standard counting of Ki-67 labeling indices in meningiomas. The automated Ki-67 LI quantisation with the use of digital tumour images ensures reliability and reproducibility of the counting results. The implementation of the new automated procedure to the diagnostic work of the neuropathologists needs to be preceded by validation on the extended studies.

Acknowledgement: This work was supported by grants: COST Action IC0604, Polish Ministry of Science and Higher Education for the years 2006-2009, the European Union in the framework of the European Social Fund through the Warsaw University of Technology Development Programme.

\section{References:}

[1] Louis DN, Oghaki H, Wiestler OD, Cavence WK (Editors). WHO Classification of Tumours of the Central Nervous System. 4th ed. Lyon: International Agency for Research on Cancer; 2007.

[2] Shiffer D. Brain Tumor Pathology: Current Diagnostic Hotspots and Pitfalls. Dordrecht: Springer; 2006.

[ 3] Kayaselçuk F, Zolrudemir S, Gümürdühü D, Zeren H, Erman T. CNA and Ki-67 in central nervous system tumors: correlation with the histological type and grade. J Neurooncol. 2002; 57(2):115-21.

[4] Pizarro CB, Oliveira MC, Coutinho LB, Ferreira NP. Measurement of Ki-67 antigen in 159 pituitary adenomas using the MIB-1 monoclonal antibody. Braz J Med Biol Res. 2004; 37(2):235-43

[ 5] Roser F, Samii M, Ostertag H, Bellinzona M. The Ki-67 proliferation antigen in meningiomas. Experience in 600 cases. Acta Neurochir. 2004;146(1):37-44.

[6] Terzi A, Saglam EA, Barak A, Soylemezoglu F. The significance of immunohistochemical expression of Ki-67, p53, p21, and p16 in meningiomas tissue arrays. Pathol Res Pract. 2008;204(5):305-14.

[7] Nakasu S, Li DH, Okabe H, Nakajima M, Matsuda M. Significance of MIB-1 Staining Indices in Meningiomas. Com- parison of Two Counting Methods. Am J Surg Pathol. 2001; 25(4):472-8.

[ 8] Soille P. Morphological image analysis, principles and application. $2^{\text {nd }}$ ed. Heidelberg: Springer; 2003

[9] Matlab Image Processing Toolbox, user's guide. The Math Works Web site. 2002. Available at: http://www.mathworks. com/applications/imageprocessing/technicalliterature.html

[10] Vapnik V. Statistical Learning Theory. $2^{\text {nd }}$ ed. New York: Wiley. 1998

[11] Schölkopf B, Smola A. Learning with Kernels. Support Vector Machines, Regularization, Optimization, and Beyond. Cambridge: MIT Press; 2002.

[12] Lezoray 0, Elmoataz A, Cardot H, Gougeon G, Lecluse M, Elie H, Revenu M. Segmentation of color images from serous cytology for automated cell classification. Anal Quant Cytol Histol. 2000;22:311-322

[13] Ruifrok AC, Katz R, Johnson DA. Comparison of quantification of histochemical staining by Hue-saturation-intensity (HSI) transformation and color deconvolution. Clin Neuropathol. 2003;11:85-91

[14] Kim YJ, Romeike BFM, Uszkoreit J, Feiden W. Automated nuclear segmentation in the determination of the Ki-67 labeling index in meningiomas. Clin Neuropathol. 2006;25(2):6773

[15] Markiewicz T, Osowski S, Patera J, Kozłowski W. Image processing for accurate recognition and counting of cells of the histological slides. Anal Quant Cytol Histol. 2006;28:281-292

[16] Markiewicz T, Wisniewski P, Osowski S, Patera J, Kozlowski W, Koktysz R. Comparative analysis of methods for accurate recognition of cells through nuclei staining of Ki-67 in neuroblastoma and estrogen/progesterone status staining in breast cancer. Anal Quant Cytol Histol. 2009;31(1):49-62.

[17] Kalala JP, Caemaert J, De Ridder L. Primary resected meningiomas: relapses and proliferation markers. In Vivo. 2004;18(4):411-16.

[18] Maillo A, Orfao A, Espinosa AB, Sayagues JM, Merino M, Sousa P, Lara M, Tabernero MD. Early recurrences in histologically benign/grade I meningiomas are associated with large tumors and coexistence of monosomy 14 and del(1p36) in the ancestral tumor cell clone. Neur Oncol. 2007;9,438-446.

[19] Rezanko T, Akkalp AK, Tunakan M, Sari AA. MIB-1 Counting Methods in Meningiomas and Agreement Among Pathologists. Anal Quant Cytol Histol. 2008;30(1):47-52.

Submitted: 15 August, 2009 Accepted after reviews: 5 November, 2009 